\title{
Histological Study on the Changes of Elastic Fibers in Localized Scleroderma Model in Thin Skin of Adult Male Albino Rat and the Possible Curative Role of Bone Marrow-Derived Mesenchymal Stem Cells
}

\author{
Maram M. Elkelany*, Amira A. Kassab, Amal A. Abd-Elhafez, \\ Amany M. Mousa and Thoraya A. El-deeb \\ Histology Department, Faculty of Medicine, Tanta University, Egypt \\ *Corresponding author
}

\section{Keywords}

Elastic fibers, Scleroderma model, Albino rat

Mesenchymal stem cells

Article Info

Accepted:

15 April 2019

Available Online:

10 May 2019
A B S T R A C T

Scleroderma is an autoimmune disease manifested by painful digital ulcers, joint contracture and skin thickening. Bone marrow-derived mesenchymal stem cells (BMMSCs) have become a recent focus of interest in tissue regeneration. The aim of this work was to study the changes of elastic fibers in localized scleroderma model in thin skin of adult male albino rat and the possible curative role of bone marrow-derived mesenchymal stem cells. 45 rats divided into control group included 15 rats, scleroderma-model group were injected with bleomycin subcutaneously in the mid back for 4 consecutive weeks, media treated group received media one day after the last bleomycin injection, stem cell treated group received suspended BM-MSCs $\left(2.5 \times 10^{6}\right)$ one day after the last bleomycin injection. Scleroderma-model and media treated groups showed black thick elastic fibers in both papillary and reticular dermis using Verhoeff's method. However, stem cell group showed black thin elastic fibers in both papillary and reticular dermis. From this study, it could be concluded that bleomycin induced structural changes in the dermis of thin skin that was ameliorated by BM-MSCs. So, it is recommended to use mesenchymal stem cells (MSCs) in scleroderma treatment.

\section{Introduction}

Scleroderma is an autoimmune disease manifested by painful digital ulcers, joint contracture, beak-shaped nose, reduced aperture of the mouth (microstomy), radial furrowing around the mouth and skin thickening. Moreover, the patient's face becomes expressionless, mask-like and appears 'mummified' (Krieg and Takehara, 2009). The term "stem cell" can be applied to group of cells that share two characteristic properties.
Firstly, they have the capacity for prolonged or unlimited self-renewal, and secondly they retain the potential to differentiate into a variety of more specialized cell types. Bone marrow stroma contains precursor cells that are capable of differentiating to hematopoietic cell and mesenchymal cell (Ryan et al., 2005). Bone marrow-derived mesenchymal stem cells (BM-MSCs) have become a recent focus of interest in tissue regeneration due to their easy isolation and culture (Chen et al., 2012). 
BM-MSCs have been found to play an important role in improving tissue fibrosis caused by various diseases such as stroke and myocardial infarction. Therefore, BM-MSCs may be considered as an attractive tool in the therapy of skin lesions (Wu et al., 2014).

Subsequently, the aim of this work was to study the changes of elastic fibers in localized scleroderma model in thin skin of adult male albino rat and the possible curative role of bone marrow-derived mesenchymal stem cells.

\section{Materials and Methods}

\section{Study design}

This study was performed on 45 adult male albino rats. They were divided into 4 groups:

Control group: It included 15 rats for histological and ultrastructural study of the thin skin.

Bleomycin-treated(scleroderma-model) group: It included 10 rats that were subcutaneously injected with $0.1 \mathrm{mg}$ of bleomycin dissolved in $0.1 \mathrm{ml}$ saline daily in the mid back for 4 consecutive weeks. Skin specimens were obtained from their mid back after 4 weeks from the last day of bleomycin injection (Juniantito et al., 2013).

Media-treated group: It included 10 rats that received subcutaneously $1 \mathrm{ml}$ of freshly prepared media after dealing as the bleomycin group.

BM-MSCs-treated group: It included 10 rats that received BM-MSCs $\left(2.5 \times 10^{6}\right)$ suspended in its specific media by subcutaneous injection in the mid back after dealing as the bleomycin group (Maria et al., 2016).

At the appropriate time, the animals were anesthetized by intraperitonial injection of sodium pentobarbital $(50 \mathrm{mg} / \mathrm{Kg})$ (Gaertner et $a l ., 2008)$ and the injected mid back skin was carefully dissected and processed for histological examination.

\section{Mesenchymal stem cells achievement}

\section{Principle}

The bone marrow of the femurs, tibias and humeri of rats were extracted, collected and cultured for mesenchymal stem cells (MSCs) achievement. When MSCs reached confluence $(70-80 \%)$ in subcultures, trypsinization was done. The cells were counted and injected at a dose of $2.5 \times 10^{6} /$ rat.

\section{For light microscopy (Verhoeff's method) (Bancroft et al., 2013)}

The specimens were immersed in $10 \%$ natural-buffered formalin for 24 hours, washed, dehydrated, cleared and paraffin immersed. Then, sections were stained using Verhoeff's method.

\section{Results and Discussion}

\section{Light microscopic results (Verhoeff's van Gieson)}

Examination of skin sections obtained from the control animals showed black colored thin elastic fibers in the reticular dermis. Elastic fibers appeared also to surround the hair follicles and sebaceous glands (Fig. 1A). However, sections of bleomycin-treated animals showed black thick elastic fibers in both papillary and reticular dermis. Moreover, thick elastic fibers were observed around the hair follicles (Fig. 1B). The same was found in specimens of bleomycin and media-treated animals (Fig. 1C). In contrast, examination of skin specimens of bleomycin and bone marrow derived-MSCs-treated animals showed black thin elastic fibers in the dermis (Fig. 1D). 
Fig.1 A photomicrograph of a section in the skin from the control group showing black, thin elastic fibers in the reticular dermis (arrows). Notice the elastic fibers surrounding the hair follicles (f) and sebaceous glands(s) (1A). A photomicrograph of a section in the skin from bleomycin-treated animal showing black thick elastic fibers in the dermis (arrows). Notice thick elastic fibers surrounding the hair follicle (f) (1B). A photomicrograph of a section in the skin from bleomycin and media-treated animal showing black thick elastic fibers in the dermis (arrows) (1C). A photomicrograph of a section in the skin from bleomycin and MSCs-treated animal showing black thin elastic fibers in the dermis (arrows) (1D) (Verhoeff's van Gieson, 6A,6B,6C,6D X 400)Control group, Bleomycin, Media, MSCs groups
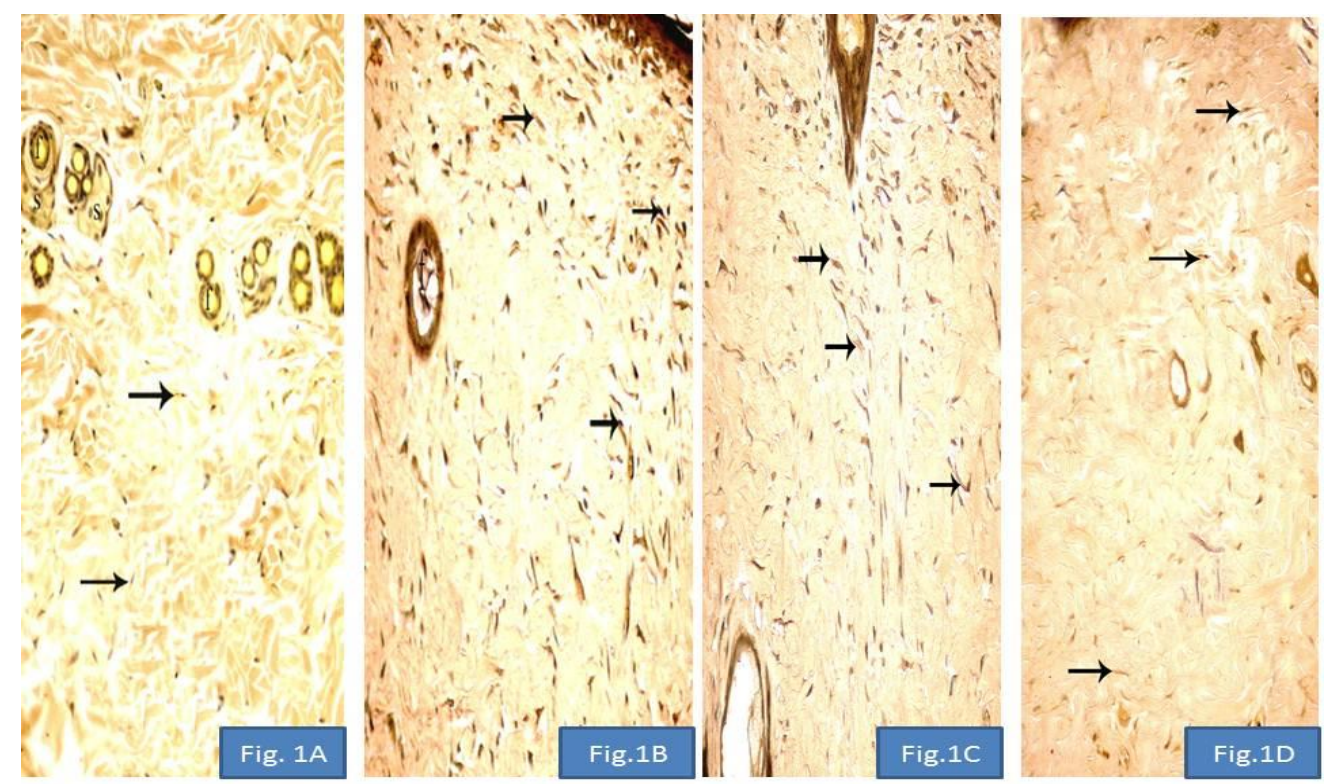

Verhoeff's Van Gieson stain of specimens of animals treated with bleomycin alone as well as bleomycin and media-treated animals of this study showed black and thick elastic fibers in both papillary and reticular dermis. This was consistent with Chatterjee et al., (2004) who observed a significant increase in the elastic fibers content in the dermis of scleroderma patients. This was confirmed by Ould-Ali et al., (2012) who observed increased density of elastic fibers during study of dermal sclerosis induced by bleomycin. This finding may be attributed to increased production of elastic fibers by the activated fibroblasts. It was stated that the pathogenesis of increased elastin deposition was caused by TGF- $\beta$ which is a potent inducer of elastin synthesis by human dermal fibroblasts.

The present work also showed thick elastic fibers that were observed around the hair follicles in Van Gieson-stained sections obtained from bleomycin-treated animals. It was stated that elastic fibers are normally present around the hair follicles (Choi et al., 2009). The increased thickness of normal elastic fibers surrounding the hair follicles may be attributed to increased production of elastic fibers. This was in agreement with Gabriela et al., (2007) who found that elastic fibers stained by orcein form a strip shape around the hair follicle in patients with scleroderma. 
Skin sections of bleomycin and MSCs-treated animals of the present work showed black thin elastic fibers in the dermis using Verhoeff's van Gieson stain which were more or less similar to the control group. The same finding was observed by Ochiai et al., (2017) during study of the role of MSCs in regeneration of skin wound. It may be attributed to the ability of MSCs to induce skin regeneration which is a limited process. Regeneration involves the gross replacement and restoration of tissue mass with normal architecture and function (Kanji \& Das, 2017).

From this study, it could be concluded that the changes of elastic fibers in localized scleroderma model can be treated by bone marrow-derived mesenchymal stem cells.

\section{References}

Bancroft, J. Christophe, L. and Kim, S. (2013): Bancroft's theory and practice of histological techniques. $7^{\text {th }}$ edition. Churchill Livingstone Elsevier. China. P. 179-202.

Chatterjee, S. Mark, M.Wooley, P. Lawrence, W. and Mayes, M. (2004): Increased dermal elastic fibers in the tight skin mouse. Clin. Exp. Rheumatol.; 22: 617-620.

Chen, J.S. Wong, V.W. and Gurtner, G.C. (2012): Therapeutic potential of bone marrow-derived mesenchymal stem cells for cutaneous wound healing. Front Immunol.; 2012: 92-103.

Choi,J. Bergdahl, A. Zheng, Q. Starcher, B. Yanagisawa, H. and Davis, E. (2009): Analysis of Dermal Elastic Fibers in the Absence of Fibulin-5 Reveals Potential Roles for Fibulin-5 in Elastic Fiber Assembly. Matrix Biol.; 28: 211-220.

Gabriela, E. Raica, M. Anca, M. and Viorica, B. (2007): Morphologic and histochemical changes in the skin of patients with scleroderma. Romanian Journal of Morphology and Embryology; 28: 361-367.

Gaertner, D.J. Hallman, T.M. Hankenson, F.C. and Batcherder, M.A. (2008): Anesthesia and Analgesia in Rodents. Anesthesia and analgesia in laboratory animals. $2^{\text {nd }}$ edition. Academic Press, San Diego, CA. Boston. P: 239-240.

Juniantito, V. Izawa, T. Yuasa, T. Ichikawa, C. Yano, R. Kuwamura, M. et al., (2013): Immunophenotypical characterization of macrophages in rat bleomycin-induced scleroderma. Veterinary pathology; 50: 76-85.

Kanji, S. and Das, H. (2017): Advances of Stem Cell Therapeutics in Cutaneous Wound Healing and Regeneration. MediatorsInflamm.; 2017: 67-79.

Krieg, T. and Takehara, K. (2009): Skin disease: a cardinal feature of systemic sclerosis. Rheumatology; 48: 14-18.

Maria, A. Toupet, K. Bony, C. Pirot, N. Vozenin, M. Petit, B. et al., (2016): Antifibrotic, Antioxidant, and Immunomodulatory Effects of Mesenchymal Stem Cells in HOClInduced Systemic Sclerosis. Arthritis Rheumatol.; 68: 1013-1025.

Ochiai, H. Kishi, K.Kubota, Y. Oka, A. Yabuki, H. Iso, Y. et al., (2017): Transplanted mesenchymal stem cells are effective for skin regeneration in acute cutaneous wounds of pigs. Regenerative Therapy; 7: 8-16.

Ould-Ali, D. Hautier, A. Andrac-Meyer, L. Bardin, N. Magalon, G. and Granel, B. (2012): Bleomycin-induced Scleroderma in nude mice can be reversed by injection of adipose tissue: evidence for a novel therapeutic intervention in systemic sclerosis. J Clin Exp Dermatol Res.; 3:164- 174. 
Ryan, J. Barry, F. Murphy, J. and Mahon, B. (2005): Mesenchymal stem cells avoid allogeneic rejection. Journal of Inflammation; 2005: 2-8.
Wu, Y. Huang, S. Enhe, J. Ma, K. Yang, S. Sun, T. et al., (2014): Bone marrowderived mesenchymal stem cell attenuates skin fibrosis development in mice. Int Wound J.; 11: 701-710.

\section{How to cite this article:}

Maram M. Elkelany, Amira A. Kassab, Amal A. Abd-Elhafez, Amany M. Mousa and Thoraya A. El-deeb. 2019. Histological Study on the Changes of Elastic Fibers in Localized Scleroderma Model in Thin Skin of Adult Male Albino Rat and the Possible Curative Role of Bone Marrow-Derived Mesenchymal Stem Cells. Int.J.Curr.Microbiol.App.Sci. 8(05): 17911795. doi: https://doi.org/10.20546/ijcmas.2019.805.207 